tsDMARD or a bDMARD in the 9 months before the start of the vaccination season (from October 1 to December 31). The observation period comprises all the years from 2006 to 2018 . Multivariate logistic regression was used to assess the association between the probability of vaccination and demographic and clinical patient's characteristics in the influenza season 2018-2019.

Results: table 1 summarizes the results. During the whole period of observation, the coverage is always under the minimum desirable threshold of $75 \%$ and far from the optimum target of $95 \%$, according to the National Italian Plan for Vaccination in the population at risk, even in the subgroup of older patients (age $\geq$ 65 years). In the logistic model including sex, age category, number and type of chronic conditions, socioeconomic status, rheumatic disease affecting the patient, area of residency, and treatment with tsDMARD or bDMARD, in 2018, older subjects were more likely to be vaccinated (people 65-74 vs 44-64, OR $4.63,95 \% \mathrm{Cl} 3.76-5.70$; people $75-84$, OR $6.61,95 \% \mathrm{Cl} 5.14-8.50$ ) as were those with diabetes comorbidity (OR 1.64, 95\%Cl 1.03- 2.59); a lower borderline-significant probability of being vaccinated was observed among patients with low socioeconomic status (OR $0.83,95 \% \mathrm{Cl} 0.68-1.00$ ), and those treated by a biologic agent (OR $0.85,95 \% \mathrm{Cl} 0.73-1.00)$

Table 1. 2006-2018 vaccination coverage in RA, PsA and AS population

2006200720082009201020112012201320142015201620172018

Global

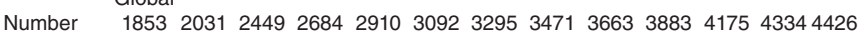
censored

Total vacci- $\begin{array}{lllllllllllll}662 & 664 & 848 & 946 & 892 & 942 & 886 & 994 & 926 & 1027 & 1232 & 1344 & 1550\end{array}$ nated (\%) (35.7) (32.7) (34.6) (35.2) (30.6) (30.5) (26.9) (28.6) (25.3) (26.4) (29.5) (31.1) (35)

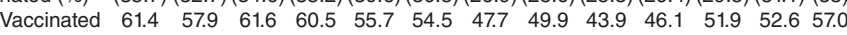
$\geq 65 \mathrm{yrs}$

(\%)

RA

$\begin{array}{llllllllllllll}\text { vaccinated } & 38.5 & 35.0 & 37.1 & 37.7 & 33.7 & 33.8 & 29.7 & 31.3 & 28.3 & 29.6 & 32.3 & 33.5 & 37.3\end{array}$ (\%)

$\begin{array}{llllllllllllll}\text { Vaccinated } & 61.9 & 56.9 & 61.1 & 59.6 & 55.2 & 54.6 & 47.4 & 49.2 & 44.3 & 46.2 & 51.5 & 51.3 & 56.0\end{array}$ $\geq 65$ yrs

(\%)

PsA

$\begin{array}{llllllllllllll}\text { vaccinated } & 28.0 & 26.6 & 29.6 & 30.8 & 24.6 & 24.6 & 23.2 & 26.0 & 21.0 & 22.3 & 26.1 & 28.8 & 32.6\end{array}$ (\%)

$\begin{array}{llllllllllllll}\text { Vaccinated } & 59.5 & 65.5 & 66.4 & 67.5 & 58.0 & 54.6 & 50.9 & 55.2 & 44.9 & 46.4 & 54.7 & 57.8 & 60.3\end{array}$ $\geq 65$ yrs

(\%)

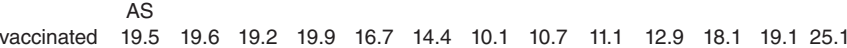
(\%)

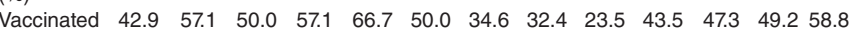
$\geq 65 \mathrm{yrs}$

(\%)

Legend: RA, rheumatoid arthritis; PsA, psoriatic arthritis; AS, ankylosing spondylitis.

Conclusion: influenza vaccination coverage is low in a population at high risk of infectious complications, even in the subgroup of elderly patients. Local guidelines are needed to improve the vaccination policies in AIIRD in order to increase the protection among patients who really need it.

References:

[1] Furer V, et al. Ann Rheum Dis 2019. Ann Rheum Dis. 2019 Aug 14. pii: annrheumdis-2019-215882

Disclosure of Interests: Luca Quartuccio Consultant of: Abbvie, Bristol, Speakers bureau: Abbvie, Pfizer, Alen Zabotti Speakers bureau: Celgene, Novartis, Janssen, Ginevra De Marchi: None declared, Tolinda Gallo: None declared, Salvatore De Vita Consultant of: Roche, GSK, Speakers bureau: Roche, GSK, Novartis, Francesca Valent: None declared

DOI: 10.1136/annrheumdis-2020-eular.2555

\section{AB1189 \\ PICASO - THE PLATFORM FOR IMPROVED PERSONAL AND COORDINATED CARE OF CHRONICALLY ILL - SIX MONTHS RESULTS FROM A PROOF-OF-CONCEPT STUDY}

J. G. Richter ${ }^{1}$, G. Chehab ${ }^{1}$, M. Tomczak ${ }^{1}$, C. Schwartz ${ }^{1}$, E. Ricken ${ }^{1}$, H. Acar ${ }^{1}$, H. Gappa ${ }^{2}$, M. Schneider ${ }^{1}$, C. Velasco ${ }^{2}$, T. P. Consortium ${ }^{2} .{ }^{1}$ Heinrich-HeineUniversity Duesseldorf, Department of Rheumatology \& Hiller Research Unit, Medical Faculty, University Hospital Dusseldorf, Duesseldorf, Germany; ${ }^{2}$ Fraunhofer-Institut für Angewandte Informationstechnik FIT, St. Augustin, Germany

Background: Cross-sectoral coordination of treatment plans and efficient management of patients with chronic diseases and co-morbidities are of great importance. In rheumatoid arthritis (RA) it is essential to orchestrate information available for a patient at various locations, to allow (cost) efficient data use, to optimize management processes and to avoid redundant diagnostics. The information and communication platform developed in the Horizon2020 funded PICASO project (www.picaso-project.eu) supports the management of patients and their data along the continuum of care, consisting of hospitals, outpatient departments, practices, other health service providers via remote health monitoring. The platform might empower patients to improve their self-management of their illnesses.

Objectives: What technological expertise and resources do RA patients and physicians have, who are willing to participate in a proof-of-concept study using a modern ICT platform? What is the user satisfaction? What are platform's clinical implications?

Methods: PICASO pursued a user-centered design approach. Platform's user requirements were determined through workshops and interviews with physicians from various disciplines, patients and other stakeholders in the health care system (e.g. data protection officers). The development was accompanied by so-called "expert walkthroughs" to ensure a user-friendly design. An evaluation concept assessing the usability of the applications, user satisfaction and clinical relevance of the platform was part of the 6-month proof-of-concept study with RA patients and their physicians (rheumatologists and family doctors). A positive ethics vote was obtained.

Results: 111 user requirements were identified and used to develop the platform. Conformity with the GDPR as well as national regulations were precisely adhered to. All developments are based on the new 'Fast Healthcare Interoperability Resources' standard enabling data exchange with other software systems in the healthcare sector. This offers many advantages, e.g. a semantic model for describing the smallest units in the health care system (e.g. medication intake times, diagnostic procedures). Thus information can be linked and made available across sectors. Data can remain with the data owner and role-specific data access is ensured.

30 RA patients ( $80 \%$ female) participated, mean age was $58.6 \pm 10.8$ years, disease duration $12.6 \pm 8.5$ years, DAS28 $2.6 \pm 0.9$, average number of comorbidities 3.0 \pm 1.6 . Patients' IT-experience was heterogeneous. After 6 months evaluations showed a good platform acceptance with an overall rating of $2.3 \pm 1.1(n=27$, Likert scale (LS) 1-6) and evaluation of 'ease of use' at 2.3 $\pm 1.2(n=27$, LS 1-6). Usability tests showed that for patients the presentation of (1) tasks to be performed fo the management of their disease, (2) results from their remote health monitoring, and (3) patient-reported outcome instruments in a dashboard was clear and easy to understand. Time required for documentation and daily tasks was rated as appropriate by $75.9 \%$ of the patients. No major technical problems or impairments due to RA where experienced when using the dashboard. 8 physicians ( $37.5 \%$ female) participated in the evaluation; overall the platform was rated at $2.2 \pm 0.5$ (LS 1-6)

Conclusion: The platform offers cross-sectoral orchestration of patient data and thus innovative capabilities for modern management processes (e.g. treat-to-target, tele-monitoring). The PICASO platform is available for RA patients as well as for other chronic diseases.

Acknowledgments: This project received funding from the European Union's Horizon 2020 research and innovation programme under grant agreement No 689209.

Disclosure of Interests: None declared

DOI: 10.1136/annrheumdis-2020-eular.1928

\section{AB1190 $\quad$ DIRECT MEDICAL COSTS OF HOSPITALIZATION DURING THE MAINTENANCE THERAPY IN PATIENTS WITH ANTINEUTROPHIL CYTOPLASMIC ANTIBODY- ASSOCIATED VASCULITIS USING JAPANESE HEALTH INSURANCE DATABASE}

R. Sakai ${ }^{1,2}$, E. Tanaka ${ }^{1}$, M. Harigai ${ }^{1} .{ }^{1}$ Tokyo Women's Medical University, Department of Rheumatology, Tokyo, Japan; ${ }^{2}$ Tokyo Women's Medical University, Division of Epidemiology and Pharmacoepidemiology, Department of Rheumatology, Tokyo, Japan

Background: Antineutrophil cytoplasmic antibody-associated vasculitis (AAV) requires a long-term maintenance therapy (MT), often accompanied by hospitalization due to relapse and/or comorbidities such as infection ${ }^{1}$. However, data about direct medical costs of hospitalization during MT in patients with AAV is limited to date despite of an increasing concern about the economic burden of patients with $\mathrm{AAV}^{2-3}$.

Objectives: To describe frequency of hospitalization and its direct medical costs during MT after the remission-induction therapy (RT) in patients with AAV using Japanese health insurance database.

Methods: This retrospective longitudinal population-based study was conducted using claims data in Japan provided by Medical Data Vision Co., Ltd. We defined individuals as AAV cases receiving RT if they met all of the following: 1) having 
at least one ICD10 code (M300, M301, M313, or M318); 2) having at least one prescription of oral corticosteroids with prednisolone-equivalent dosage $\geq 30 \mathrm{mg}$ / day, methylprednisolone pulse therapy, immunosuppressive drugs (cyclophosphamide [IVCY], methotrexate, or mycophenolate mofetil), or rituximab (RTX) during hospitalization between April 2008 and April 2017; and 3) having at least 7 days of hospitalization. The observation started from the next day of discharge from the first hospitalization for RT and ended at 24 months later, the month of loss of follow-up, or April 2017. We described the frequency of hospitalization and calculated direct medical costs (per month) during the observation. We analyzed medical costs from a societal perspective. We classified reasons of hospitalization into 3 categories; intensification of treatments for AAV, AAV MT including IVCY or RTX treatments, and comorbidities (infection, cardiovascular disease [CVD], malignancy, and others) using ICD10 codes plus treatments or interventions during the hospitalization.

Results: In this study, 1,703 patients with AAV were included. The median [IQR] age was $72[63,79]$ years and $55.7 \%$ were female. The total number of hospitalization was 1,897 in 863 patients $(50.7 \%)$. Among the hospitalizations, 296 hospitalization in 235 patients were categorized as intensification of treatments for AAV, 627 hospitalization in 297 patients were AAV MT, and 974 hospitalization in 572 patients were categorized as comorbidities. In the last category, infections were most frequent (220), followed by malignancy (54) and CVD (15). The mean direct medical costs per month was 20,945 EUR (1 EUR=125 JPY) in patients with hospitalization and 599 EUR in those without. Patients with hospitalization due to intensification of treatments for AAV had the highest direct medical costs (3,000 EUR), followed by those with hospitalization due to comorbidities $(2,001$ EUR), and those with hospitalization due to AAV MT (1,649 EUR).

Conclusion: More than half of the patients had hospitalization during MT, and hospitalization due to comorbidities were most frequent. The mean direct medical costs in patients with at least one hospitalization was approximately 3.5 times as high as that in those without hospitalization.

References:

[1] Presse Med. 2015; 44:e251-e257

[2] J. Rheumatol. 2015; 42:2383-91

[3] Clin Exp Rheumatol.2019:137-43

Acknowledgments: This work was supported by AMED under Grant Number JP17ek0109121.

Disclosure of Interests: Ryoko Sakai Grant/research support from: Tokyo Women's Medical University (TWMU) has received unrestricted research grants for Division of Epidemiology and Pharmacoepidemiology of Rheumatic Diseases from Ayumi Pharmaceutical Co. Ltd., Bristol Meyers Squib, Chugai Pharmaceutical Co. Ltd., Nippon Kayaku Co. Ltd., Taisho Toyama Pharmaceutical Co. Ltd. Mitsubishi Tanabe Pharma Corp., and with which TWMU paid the salary of R.S. Eiichi Tanaka Consultant of: ET has received lecture fees or consulting fees from Abbvie, Asahi Kasei pharma co., Bristol Myers Squibb, Chugai Pharmaceutical, Daiichi Sankyo Co., Eisai Pharmaceutical, Janssen Pharmaceutical K.K., Nippon Kayaku, Pfizer, Takeda Pharmaceutical, Taisho Toyama Pharmaceutical Co., and UCB Pharma., Speakers bureau: ET has received lecture fees or consulting fees from Abbvie, Asahi Kasei pharma co., Bristol Myers Squibb, Chugai Pharmaceutical, Daiichi Sankyo Co., Eisai Pharmaceutical, Janssen Pharmaceutical K.K., Nippon Kayaku, Pfizer, Takeda Pharmaceutical, Taisho Toyama Pharmaceutica Co., and UCB Pharma., masayoshi harigai Grant/research support from: AbbVie Japan GK, Ayumi Pharmaceutical Co., Bristol Myers Squibb Co., Ltd., Eisai Co. Ltd., Mitsubishi Tanabe Pharma Co., Nippon Kayaku Co., Ltd., and Teijin Pharma Ltd. $\mathrm{MH}$ has received speaker's fee from AbbVie Japan GK, Ayumi Pharmaceutical Co., Boehringer Ingelheim Japan, Inc., Bristol Myers Squibb Co., Ltd., Chuga Pharmaceutical Co., Ltd., Eisai Co., Ltd., Eli Lilly Japan K.K., GlaxoSmithKline K.K., Kissei Pharmaceutical Co., Ltd., Oxford Immuotec, Pfizer Japan Inc., and Teijin Pharma Ltd. MH is a consultant for AbbVie, Boehringer-ingelheim, Kissei Pharmaceutical Co., Ltd. and Teijin Pharma.

DOI: 10.1136/annrheumdis-2020-eular.806

\section{AB1191 AWARENESS OF PRESCRIPTION DRUGS FOR RHEUMATOID ARTHRITIS AMONGST PATIENTS -A COMPARISON OF THE RESULTS FROM 2014 AND 2018 SURVEYS-}

M. Sato ${ }^{1}$, M. Takemura ${ }^{2} .{ }^{1}$ Kaizu Medical Association Hospital, Rheumatology, Kaizu City, Gifu, Japan; ${ }^{2}$ Fujita Health University, Advanced Diagnostic System Research Laboratory, Toyoake, Aichi, Japan

Background: Treatment of rheumatoid arthritis (RA) is based on drug therapy. With the increasing number of effective drugs being authorized for use and generic drugs becoming available in the market, patients with RA now have an abundance of drugs as treatment options.

Objectives: To conduct a survey of RA patients to evaluate their knowledge about the prescribed drugs, their names, and the respective categories.

Methods: In 2014 and 2018, two different surveys were done in which RA patients were interviewed regarding the name of biologics (trade name) and other oral medications (category and trade name of anti-rheumatic drugs, steroid drugs and anti-inflammatory analgesics). The results of the two investigations are compared in this study.

Results: A total of 135 (34 men and 101 women) and 184 patients ( 31 men and 153 women) were interviewed in the surveys done in 2014 and 2018, respectively. In the 2014 survey, the mean age of the patients was 58.5 years (range: 25-88 years), while in the 2018 survey, the mean age of the patients was 61.0 years (range: 14-84 years). The various biologics prescribed to the patients who participated in the 2014 vs. 2018 surveys were as follows: infliximab (27 vs. 22), etanercept (11 vs. 9), adalimumab (14 vs. 16), tocilizumab (43 vs. 71 ), abatacept (29 vs. 46 ), golimumab (7 vs. 11 ), certolizumab-pegol (4 vs. 3 ), sarilumab (0 vs. 2), and tofacitinib (0 vs. 5), respectively. The number of patients who were prescribed various categories of oral medications, as stated in the 2014 vs 2018 surveys, was as follows: anti-rheumatic drugs, $104(77.0 \%)$ vs. $131(71.2 \%)$ steroid drugs, $36(26.7 \%)$ vs. 44 (23.9\%); and anti-inflammatory analgesics, 49 $(36.3 \%)$ vs. $61(33.2 \%)$, respectively. The number of patients that took medications without any knowledge about the drug name or its category, as reported in the 2014 vs. 2018 surveys was as follows: anti-rheumatic drugs, 24 (23.1\%) vs. $42(32.1 \%)$; steroid drugs, $11(30.1 \%)$ vs. 24 (54.5\%); and anti-inflammatory analgesics, $15(30.6 \%)$ vs. 17 (27.9\%), respectively. In the corresponding years the number of patients who responded negatively to the question whether they knew about the trade name of the biologics prescribed to them was $15(11.1 \%)$ and 26 (14.1\%), in the 2014 and 2018 surveys, respectively. The mean age of the patients who expressed lack of knowledge with respect to the trade name of the biologics prescribed to them was 67.3 and 69.5 years old, in the 2014 and 2018 surveys, respectively; thus suggesting the impact of old age on awareness about prescribed drugs. Many of the biologics prescribed to these patients were intravenous drip formulations, and only one patient was prescribed self-administered subcutaneous injection formulation. This implied that the majority of patients who expressed lack of knowledge regarding the trade name of the biologics were administered the drug by health-care providers at the hospital.

Conclusion: Our investigation about RA patients' understanding of the trade names and category of the drugs they were administered revealed that 20 to $50 \%$ of the patients were unaware about the oral medications they were receiving. In particular, there were many patients who had misinterpreted steroidal drugs as analgesics. In addition, approximately $10 \%$ of the patients lacked an understanding of drugs that require cautious use due to their potential for causing adverse events. For those biologics administered at the hospital by healthcare providers, the patients had a lack of inclination to learn the drug name. In today's era, with the emergence of generic drugs and an increase in the drug categories, it is not easy for the aging patients to understand and remember information about the prescription drugs. Hence, it is necessary to come up with measures to tackle this situation.

Disclosure of Interests: None declared

DOI: $10.1136 /$ annrheumdis-2020-eular. 1378

\section{AB1192 THE UNDERWORLD OF DEPRESSIVE SYMPTOMS IN RHEUMATIC DISEASES: OVERLOOKED, UNRECOGNIZED OR UNPERCEIVED?}

F. Ingegnoli ${ }^{1,2}$, T. Schioppo $^{1,2}$, T. Ubiali ${ }^{1}$, V. Bollati ${ }^{2,3}$, S. Ostuzzi ${ }^{4}$, M. Buoli $^{2,5}$, R. Caporali ${ }^{1,2} .{ }^{1} \mathrm{G}$ Pini Hospital, Clinical Rheumatology Unit, Milan, Italy; ${ }^{2}$ Università degli Studi di Milano, Milan, Italy; ${ }^{3}$ EPIGET Lab, Milan, Italy; ${ }^{4}$ ALOMAR Lombard Association for Rheumatic Diseases, Milan, Italy; IRCCS Ca'Granda Policlinico, Milan, Italy

Background: The concomitant presence of depressive symptoms and rheumatic diseases (RDs) impose a considerable economic and social burden on the communities as they are associated with numerous deleterious outcomes such as increased mortality, work disability, higher disease activity and worsening physical function, higher pain levels and fatigue. Despite growing interest on depressive symptoms burden in RDs, current patient perception on this topic is unknown.

Objectives: Italian patients with RDs were invited to participate in an online study gauging the presence and the perception of depressive symptoms using the Patient Health Questionnaire (PHQ-9).

Methods: This was a cross-sectional no-profit online study to screen the presence and the perception of depressive symptoms in RDs patients. All participants gave their consent to complete the PHQ-9 and they were not remunerated Completion was voluntary and anonymous. The PHQ-9 rates the frequency of symptoms over the past 2 weeks on a 0-3 Likert-type scale. It contains the following items: anhedonia, depressed mood, trouble sleeping, feeling tired, change inappetite, guilt or worthlessness, trouble concentrating, feeling slowed down or restless, and suicidal thoughts. Patients were stratified as: $<4$ not depressed, 5-9 sub-clinical or mild depression, 10-14 moderate depression, 15-19 moderately severe depression and 20-27 severe depression. The survey was disseminated by ALOMAR (Lombard Association for Rheumatic Diseases) between June and October 2019 